\title{
Des expertises scientifiques crédibles en appui à la décision et au débat publics. Retour d'expérience sur les ESCo de l'Inra
}

\author{
Claire Sabbagh ${ }^{1}$, Yves Le Bars $^{2}$, Pierre Stengel ${ }^{3}$ \\ ${ }^{1}$ Ingénieur responsable de l'activité d'expertise collective scientifique à l'Inra, Inra, 75007 Paris, France \\ ${ }^{2}$ Gestionnaire de la recherche en ingénierie de l'agriculture et de l'environnement, 92160 Antony, France \\ ${ }^{3}$ Agronome, Inra, 75007 Paris, France
}

\section{Introduction}

L'émergence croissante de questions d'intérêt collectif, marquées par des controverses sociétales et la défiance entre les parties prenantes concernées, induit une demande accrue d'éclairages scientifiques. Elle émane particulièrement des pouvoirs publics en attente de fondements justifiant leurs actions. Face à cette demande, l'expertise scientifique fait l'objet de critiques. Sa mise en cause la plus fréquente porte sur l'indépendance des chercheurs et des institutions intervenant dans l'expertise à l'égard des intérêts en jeu. Elle atteint plus globalement la légitimité d'une expertise basée sur la seule science, dont la portée est contestée en raison de l'incomplétude de la connaissance. Cette situation suscite des propositions visant à lui substituer des procédures mixtes d'expertise, associant des détenteurs de savoirs "profanes » aux chercheurs spécialisés. L'Inra (Institut national de la recherche agronomique), organisme de recherche finalisée dans les domaines de l'agriculture, de l'alimentation et de l'environnement, doit répondre à l'attente d'expertise des décideurs publics sur des questions stratégiques dans un contexte globalisé : changement climatique, sécurité alimentaire, pressions sur les ressources naturelles... Son choix a été d'organiser sa capacité d'expertise par la mise en place d'un cadre institutionnel défini par une "charte de l'expertise scientifique ", avec la création d'une structure d'appui et d'animation, la Délégation à l'expertise scientifique, à la prospective et aux études (DEPE). Dans ce cadre, sept expertises scientifiques collectives (ESCo) ont été produites, de 2002 à 2010.

Le défi consiste à apporter aux décideurs publics une expertise scientifique crédible dans la société, ce qui suppose d'assurer la propre légitimité de l'Institut comme producteur de cette expertise. L'objectif de l'Inra a donc été, d'une part, de garantir au mieux l'indépendance du processus de réalisation et la compétence des experts impliqués, d'autre part, de limiter les assimilations entre expertise et décision en renonçant à toute recommandation.

\footnotetext{
Auteur correspondant : C. Sabbagh, claire_sabbagh@yahoo.fr
}

Claire Sabbagh a été directrice de l'unité Expertise scientifique collective à l'Inra de 2002 à 2010, directrice adjointe de la Délégation à l'expertise, à la prospective et aux études (DEPE) de 2010 à 2012 ; elle est membre du Comité de déontologie et de prévention des conflits d'intérêts à l'Agence nationale de sécurité sanitaire de l'alimentation, de l'environnement et du travail (Anses).

Yves Le Bars est ingénieur général honoraire du génie rural, des eaux et des forêts. Il a dirigé pendant une vingtaine d'années des organismes de recherche finalisée. Il a été président du comité d'orientation de l'étude Écophyto R\&D (2007-2009) - Quelles voies pour réduire l'usage des pesticides -, chargé, en lien avec les coordinateurs des groupes d'experts, de garantir le bon déroulement du processus et d'assurer l'apprentissage mutuel entre tous les acteurs. Il est président du conseil scientifique de l'Isara-Lyon (Institut supérieur d'agriculture Rhône-Alpes), et depuis 2012, président du Comité français pour la solidarité internationale (CFSI). Pierre Stengel a été directeur scientifique « Environnement, écosystèmes cultivés et naturels » à l'Inra, responsable de l'unité Expertise scientifique collective et membre du Collège de direction de l'Institut (2001-2009). 
Encadré 1. L'expertise scientifique collective en appui aux politiques publiques à $l^{\prime}$ Inra

L'expertise figure dans les missions nationales pour la recherche publique et ses chercheurs (au sens de l'article 411 du code de la recherche). Pour l'Inra, une ESCo répond à une commande publique. Elle est régie par une charte de l'expertise qui énonce 4 principes : compétence, impartialité, pluralité, transparence. La complexité des questions posées exige un traitement pluridisciplinaire. L'objectif est de réaliser un état des connaissances publiées qui dégage les consensus, pointe les incertitudes, les controverses et les lacunes. Une ESCo ne comporte ni avis ni recommandation.

En 2012, deux évaluations de ces ESCo ont été réalisées, sur la base d'interviews de chercheurs et cadres scientifiques de l'Inra. La première, confiée à Pierre Stengel, directeur scientifique à l'Inra, responsable de l'activité d'expertise collective, a porté sur les conditions de la mobilisation des chercheurs. La seconde, conduite par un observateur extérieur, Yves Le Bars, a traité des retombées de l'expertise sur le travail de recherche. Cet article propose une synthèse des éléments issus de ces évaluations, fruit de discussions entre les auteurs et Claire Sabbagh, alors en charge de la conduite des expertises collectives à l'Inra ; ces éléments font converger le témoignage des experts, l'expérience de praticiens en conduite d'expertise et un regard averti mais libre de parti pris institutionnel. Trois questions-clés ressortent de cette réflexion : la question de l'indépendance qui fonde la crédibilité de l'expertise, celle de sa pertinence et enfin celle de son appropriation par ses utilisateurs potentiels, commanditaires, chercheurs et parties prenantes.

\section{L'indépendance de l'expertise et les conflits d'intérêts : de l'indépendance de l'expert à l'impartialité de l'expertise}

\section{Les experts et les intérêts économiques}

C'est le point le plus discuté aujourd'hui où nombre d'expertises sont mises en cause du fait de liens découverts a posteriori entre un expert et des intérêts privés. Les agences d'expertise, en France et en Europe, ont mis en place des dispositifs de déclarations systématiques d'intérêt (DCI). Ces intérêts s'apprécient au niveau des individus - fonctions de conseil auprès de structures privées - et des collectifs de recherche - contrats, prestations, bourses. À l'Inra, les experts sont initialement repérés sur la base de leur compétence, avérée par leurs publications scientifiques. La DCI permet d'assurer la transparence des liens que les experts pressentis pourraient avoir noués avec des structures privées, en s'interrogeant sur les biais que ces liens pourraient entraîner dans l'objectivité attendue de l'analyse et en écartant éventuellement les personnes jugées trop proches d'intérêts spécifiques.

\section{Des intérêts d'autres natures et moins identifiables}

L'existence d'intérêts privés apparaissant comme le principal obstacle à l'impartialité de l'expertise, on pourrait supposer qu'une fois cet obstacle soumis à la règle de transparence et contrôlé, l'expertise écarterait suffisamment les risques de parti pris pour s'approcher d'une analyse strictement scientifique des sources. Cette hypothèse repose sur une conception de la science fondée sur deux postulats :

- la neutralité axiologique de la science, la Wertfreiheit de Max Weber, souvent invoquée pour justifier l'image d'un savant libre de passions partisanes ;

- la radicale opposition entre les faits qui caractérisent la démarche scientifique et les valeurs qui déterminent les choix pour l'action. La science dirait ce qu'il en est du réel pour un observateur objectif, les valeurs déterminant les décisions politiques.

Or, dans la réalité, les connaissances sont portées par des chercheurs qui ne sont pas déconnectés des conditions dans lesquelles sont produites ces connaissances (financement, partenariat, écoles de pensée...) et qui ne forment pas une communauté homogène spontanée d'intérêts, de cultures, de points de vue sur les questions posées. Chaque scientifique expert est porteur d'une vision de la problématique qui lui est soumise et, très souvent, en posture de défense ou de promotion de son domaine de recherche. Le souci de démontrer la pertinence de ce dernier vis-à-vis de grands enjeux collectifs, que l'expertise peut ou non valider, peut primer sur le recul critique attendu. Il est particulièrement prégnant dans les domaines de recherche marqués par de tels enjeux comme l'environnement ou l'alimentation. Les experts en science du sol ont, par exemple, éprouvé une véritable déception en mesurant la faible efficacité d'actions d'amélioration du bilan national de $\mathrm{CO}_{2}$ par stockage de carbone dans le sol.

Ainsi, la notion de conflits d'intérêts dans sa définition classique ne rend pas compte d'autres formes de dépendance "invisibles ", plus délicates à repérer. Une discipline scientifique est en effet associée à une culture, qui inclut un socle commun de lois, de théories, d'instruments et de vocabulaire, mais aussi des relations sociales avec des acteurs extérieurs à la recherche (revues scientifiques, partenaires). Les agronomes, par exemple, n'ont pas la même approche de la notion de biodiversité que des écologues, les toxicologues n'ont pas le même regard 
sur l'emploi des pesticides que des chercheurs en phytopharmacie, un économiste n'a pas, sur la question de l'utilisation de l'eau en agriculture, le même cadre d'analyse qu'un spécialiste de l'irrigation des cultures. Chaque discipline se réfère à ce que Thomas Kuhn appelle «la science normale », au sens où celle-ci fonctionne sur un système cohérent de vision du monde qui repose sur un cadre théorique ou une école de pensée. La compétence de l'expert est nécessairement bornée par son horizon de connaissances, par les partis pris épistémologiques et philosophiques propres à sa discipline et à son parcours. Or il est attendu de l'expertise qu'elle fasse état de la diversité des hypothèses en cours. Il faut, dans la constitution du collège d'experts, être attentif aux représentations que les chercheurs se font de la question posée en fonction de leur appartenance disciplinaire. Les évolutions récentes des pratiques d'expertise montrent que l'accent s'est déplacé de l'objectif d'indépendance des experts, dont on perçoit les limites, à celui d'impartialité du processus d'expertise, fondée sur des procédures de conduite structurées par des principes, des savoir-faire et des méthodes.

\section{Positionnement et intérêts institutionnels}

L'aide à la décision fait partie des missions des organismes de recherche qui doivent apporter des éléments concrets à la résolution de problèmes rencontrés par les utilisateurs. À l'Inra, une part importante des recherches est réalisée avec des partenaires professionnels de l'agriculture, des industries agroalimentaires et/ou avec le monde associatif. La conséquence est l'existence fréquente de liens d'intérêts des experts et, sans doute plus insidieusement, une imprégnation collective des enjeux et points de vue des parties prenantes concernées.

Les interactions nécessaires avec les acteurs dans le champ de l'agriculture, de l'environnement et de l'alimentation, sont une source de pertinence mais aussi un risque de biais pour l'expertise. Cette difficulté atteint inévitablement l'institution elle-même. En raison du risque de mettre en péril les intérêts socioéconomiques portés par ses partenaires, l'Inra peut faire l'objet de doutes quant à son engagement dans le traitement objectif de questions dérangeantes pour la sphère agricole. Fonder sa légitimité à traiter de la réduction d'usage des pesticides ou de la douleur des animaux d'élevage, en considérant des techniques plus respectueuses, mais aussi moins productives, impose une grande vigilance pour attester une double indépendance collective des experts : indépendance à l'égard d'une culture d'établissement implicite, indépendance à l'égard de toute pression hiérarchique.
Les réponses apportées par l'Inra pour neutraliser ces effets ont été de plusieurs natures. La première est la pluridisciplinarité du groupe d'experts et son ouverture impérative à des appartenances institutionnelles différentes. Quant au contrôle institutionnel, il a été strictement limité à la vérification du respect de la procédure et de la charte interne de l'expertise. La conformité des contenus du rapport et de sa synthèse vis-à-vis des sources bibliographiques en est un élément majeur. Le "pilote scientifique», qui conduit le travail du collège d'experts avec les responsables des chapitres du rapport, garantit que les documents publiés sont fidèles à la production des experts et prémunit d'un hypothétique risque de censure. À cet égard, des experts extérieurs à l'Inra disent avoir perçu le poids de la « culture d'établissement » dans le collège, mais n'ont pas ressenti d'intervention institutionnelle préjudiciable à leur propre expression.

\section{Un cadre déontologique et méthodologique soutenant l'impartialité}

La conduite d'une ESCo obéit à l'Inra à des principes énoncés dans une charte et déclinés dans un code de procédures qui décrit les différentes étapes du processus, de la lettre de commande à la restitution des conclusions du travail. La délégation à l'expertise (DEPE) rassemble des spécialistes de la réalisation de ce processus. Ils appuient les experts et veillent au respect des règles. Un directeur scientifique de l'Institut exerce en son nom la responsabilité de maître d'ouvrage. Il veille particulièrement à la maîtrise des relations avec les commanditaires de l'ESCo: respect des termes de la commande par les experts, respect de l'indépendance des experts par les commanditaires. Cet engagement institutionnel fort garantit que l'acceptation de la commande d'expertise par l'Inra est associée à l'acceptation de sa charte par le commanditaire. L'encadrement déontologique et méthodologique qui pose le caractère reproductible de l'exercice, la rigueur et la transparence de sa réalisation sont les éléments-clés de l'indépendance du processus et de la qualité de son résultat.

\section{Thèmes d'expertises et pluralité}

La spécificité des ESCo est qu'elles ne traitent pas de questionnements étroitement ciblés sur un produit, une pratique ou une discipline. Elles concernent un domaine de l'action publique, avec des thématiques larges, comme « Agriculture et sécheresse » ou « Agriculture et biodiversité ». Ces questions sont traversées par des controverses sociotechniques (impacts des pesticides, statut des animaux, tensions entre agriculture et biodiversité...) qui occupent une place centrale dans le débat public. Un des 
intérêts d'une ESCo est de vérifier si la controverse de société trouve ou non un écho dans l'état de la science. L'analyse critique de la littérature publiée doit conduire à établir un état contrasté des connaissances, permettant aux décideurs de distinguer les noyaux durs de savoirs, qui peuvent fonder la décision, et les éléments plus fragiles ou émergents qui suggèrent prudence et précaution. La pluralité des disciplines et des points de vue est recherchée dans la constitution du collège d'experts. L'ESCo « Pesticides » rassemblait des experts en écologie, agronomie, physiologie et génétique végétale, économie, sociologie. Le collège pour la douleur animale comprenait un médecin, spécialiste de la douleur humaine, des physiologistes, des généticiens, des éthologues, des vétérinaires et des spécialistes des sciences humaines (histoire, anthropologie, philosophie, droit). La pluralité dans le collège d'experts s'exprime également par une diversité d'approches, de cultures, de points de vue. La règle est de recruter 30 à $50 \%$ des experts à l'extérieur de l'Institut dans des établissements de recherche en France (CNRS, Inserm, Universités, IRD, Irstea...) et à l'étranger (Canada, Suisse, Belgique...).

La pluralité présente l'avantage de neutraliser les partis pris et de faire s'exprimer, à travers le débat, les accords et désaccords entre spécialistes. La démarche n'est pas sans risque. Elle suppose, dans la phase d'assemblage des «briques de base » disciplinaires, de s'aventurer hors des champs académiques strictement balisés, pour donner une interprétation collective des faits scientifiques en éclairant les questions posées. La pluralité y joue un rôle majeur dans la discussion des hypothèses, la construction et la validation d'une synthèse argumentée.

\section{Les conditions de la pertinence de l'expertise}

\section{La capacité à construire les questions scientifiques pour éclairer la décision}

L'expertise met en présence deux mondes différents, le politique et le scientifique, dont les logiques doivent se rencontrer sans se confondre. Sa première étape consiste à traduire les questions de la société - emploi des pesticides, traitement des animaux d'élevage, santé et alimentation - en éléments de connaissances à mobiliser pour éclairer la décision ${ }^{1}$.

La difficulté de la science à se saisir des questions posées par la société tient d'abord au fait que les problématiques scientifiques ne recoupent pas en général les domaines de l'action publique. Chaque discipline

\footnotetext{
1 Roqueplo, P., 1999. Entre savoir et décision, l'expertise scientifique, Versailles, Quæ.
}

Encadré 2. Les 7 ESCo menées à l'Inra (2002-2010) et évaluées en $2012^{2}$

- Stocker du carbone dans les sols agricoles de France (2002)

- Pesticides, agriculture et environnement (2005)

- Sécheresse et agriculture (2006)

- Les fruits et légumes dans l'alimentation (2007)

- Agriculture et biodiversité (2008)

- Douleurs animales (2009)

Les comportements alimentaires (2010)

revendique son autonomie pour se poser les «bonnes » questions de recherche et récuse celles qu'elle ne juge pas pertinentes. "Une discipline scientifique, écrit Heinz Wismann, se caractérise par une autolimitation volontaire par rapport au projet philosophique et donc par une efficacité heuristique et une légitimité épistémologique autre ${ }^{3}$.» Les connaissances nécessaires pour éclairer la décision sont en outre distribuées entre un grand nombre de disciplines et ne constituent pas un corpus organisé qu'on pourrait facilement regrouper pour apporter des réponses ajustées aux questions posées. Pour certains chercheurs interviewés, la conscience de cette limite peut être dissuasive: ils ne perçoivent pas la pertinence de leurs connaissances visà-vis des réponses à produire par l'expertise.

\section{Une phase préalable d'instruction des questions posées à l'expertise}

Cette phase initiale de l'expertise consiste à créer les conditions d'une compréhension claire des questions posées par les commanditaires, replacées dans leur contexte et situées par rapport à leurs enjeux. Elle se caractérise par des itérations entre les commanditaires (ministère de l'Agriculture, ministère de l'Écologie, principalement) et l'Inra et permet de problématiser la préoccupation du politique en questions que les connaissances scientifiques pourront éclairer. Ce processus aboutit à la délimitation, et éventuellement à la restriction du champ de l'ESCo, justifiée par la disponibilité des sources bibliographiques et la faisabilité de l'expertise dans les délais impartis. Un cahier des charges qui a valeur contractuelle en formalise les termes. Cet ajustement aux

\footnotetext{
2 Pour en savoir plus sur ces ESCo et notamment pour télécharger les synthèses, consulter le site internet de la DEPE : http:/ / www6.paris.inra.fr/depe/Presentation/L-expertisescientifique-collective.

3 Wismann, H., 2010. Au-delà du scientisme, in Besnier, J.-M., Klein, É., Le Guyader, H., Wismann, H. (Eds), La science en jeu, Actes Sud /IHEST, 41-60.
} 
Encadré 3. Le déroulement d'une ESCo

- Durée : 18 mois à 2 ans

- 20 à 25 experts emmenés par des pilotes scientifiques, assistés par une équipe projet chargée de l'organisation

- Déroulement

1. Phase d'instruction de la question

2. Constitution du corpus bibliographique

3. Constitution du collège d'experts, travail d'extraction et d'analyse des éléments pertinents de la bibliographie

4. Assemblage des contributions individuelles

5. Rédaction du rapport et de la synthèse

6. Présentation et diffusion des résultats

besoins fondateurs de la commande, pour assurer la cohérence et l'efficacité globale de l'expertise, est à la fois délicat et déterminant pour la suite.

\section{Le matériau bibliographique, au fondement de l'expertise}

\section{Évaluer la disponibilité des connaissances}

Le principe général d'une ESCo est d'exploiter la littérature scientifique certifiée par les pairs. Or les thèmes d'expertise relèvent souvent de problématiques en cours de construction. La littérature, même abondante, est d'abord dédiée à les fonder factuellement et conceptuellement. Les travaux sur la maîtrise et la gestion sont généralement postérieurs. Accepter une demande d'ESCo suppose au préalable une évaluation du corpus bibliographique qui permet de mesurer l'ampleur des références à traiter. Une extrême abondance pourra conduire à restreindre le champ des questions. À l'inverse, la demande peut être refusée si les connaissances sont insuffisantes.

\section{La constitution du corpus, une phase essentielle}

Une ESCo suppose un inventaire complet des ressources documentaires disponibles. Le corpus bibliographique a une triple fonction :

i) permettre le repérage des experts ;

ii) identifier les disciplines à mobiliser pour traiter la question ;

iii) repérer le matériau sur lequel vont travailler les experts.
L'Inra s'engage sur la production d'une analyse systématique de la littérature scientifique. La qualité du corpus, issu des bases de données internationales, dépend de la pertinence des mots-clés et des équations de recherche documentaire. Cette étape comporte des risques de "passer à côté " de références intéressantes que les mots-clés n'auraient pas permis de capturer. Dans l'ESCo «Douleurs animales», par exemple, l'absence initiale du mot-clé "conscience animale » a conduit à ne pas intégrer d'emblée les études des sciences cognitives qui formulent des hypothèses sur des états de conscience chez les poissons et invertébrés, jusqu'ici réputés ne pas ressentir la douleur. Leur prise en compte en cours de travail a permis d'ouvrir un débat sur la caractérisation d'états de douleur en fonction des espèces et a amené à formuler des besoins de connaissances complémentaires.

\section{Un recul critique indispensable pour l'animation de l'expertise}

L'indépendance du processus par rapport aux intérêts et aux partis pris suppose une capacité d'analyse fine des situations d'expertise. Quels sont les intérêts et positions en présence et ont-ils des échos dans les communautés scientifiques? Quels équilibres introduire dans le groupe d'experts pour neutraliser des partis pris ? Comment s'assurer que le corpus fait état de la diversité des hypothèses et des approches ?

L'animation des groupes d'experts implique une capacité de recul pour mettre à jour les domaines de savoirs pertinents, pour appréhender le collège d'experts non pas comme une entité globale mais comme un ensemble de chercheurs porteurs non seulement de connaissances mais aussi de valeurs, pour reformuler à chaque étape les avancées du travail, et enfin pour produire les documents de sortie sous une forme accessible aux utilisateurs. Cette fonction de médiation est confiée à l'Inra à la DEPE, composée principalement d'ingénieurs possédant une solide culture générale, des compétences en information scientifique et technique et en conduite de projets. L'équipe en charge d'une ESCo a, entre autres, pour tâche de maîtriser plusieurs risques inhérents au fonctionnement interne du groupe d'experts :

- l'autocensure : l'expert renonce à exprimer son avis, le sachant minoritaire ou susceptible de le couper du groupe ;

- l'exclusion non justifiée d'hypothèses scientifiques portées par d'autres écoles de pensée ;

- le gentleman's agreement entre experts qui, par crainte de voir relativisé le savoir scientifique, préfèrent confiner la controverse à la sphère de la communauté des pairs ;

- la domination de certaines disciplines, liée à leur ancienneté, à leur notoriété, qui doit être contenue pour 
laisser s'exprimer des disciplines considérées comme moins armées et pourtant légitimes pour traiter des questions posées.

\section{L'appropriation des expertises, bénéfices scientifiques et utilité sociale}

\section{Faire de l'expertise une ressource publique}

Les premiers destinataires d'une ESCo sont ceux qui l'ont commandée et qui souhaitent l'utiliser comme support à leurs décisions. Mais un facteur-clé de reconnaissance pour l'expertise scientifique est qu'elle soit rendue publique et permette à toutes les parties prenantes, et, au-delà, à tout citoyen intéressé, de s'en saisir, de l'exploiter au bénéfice de ses propres actions et de la critiquer. Les restrictions dans l'accès aux produits de l'expertise et à la connaissance de ses modalités de réalisation doivent faire l'objet d'une vigilante prévention. Dans le cas d'une ESCo, les moyens de diffusion sont: i) la rédaction, sous le contrôle du groupe des experts, d'une synthèse que son volume (une quarantaine de pages) et sa forme rendent accessible à un public non spécialisé ; ii) la production d'un 4 pages reprenant les principales conclusions ; iii) la tenue d'un colloque rassemblant parties prenantes et médias.

La diffusion large d'un état de l'art scientifique portant sur un enjeu sociétal induit des réactions des parties prenantes qui y identifient les arguments favorables ou défavorables à leur propre cause. Le débat sous-jacent peut ainsi se réduire dans un premier temps à une opposition pro versus anti-expertise. Cette phase peut être durable à hauteur de l'importance des enjeux. Le cas du GIEC l'illustre manifestement. Au-delà, la mise à disposition des connaissances et de leurs limites doit être considérée par chaque porteur d'enjeu comme une ressource qu'il mobilise pour s'adapter à l'évolution consécutive du débat public. L'expertise trouve alors sa juste place dans les processus collectifs d'évaluation et de fabrication des décisions.

\section{L'appropriation par la recherche}

Si l'appropriation de l'expertise par l'action publique est l'objectif premier, il est moins courant de s'interroger sur ses retombées pour la recherche. Pour l'Inra, une ESCo constitue un moyen de valoriser à la fois ses compétences et les connaissances produites. Au-delà, elle constitue un support d'analyse critique des orientations et des questionnements des recherches. La synthèse scientifique qu'elle opère permet de stabiliser les consensus, de repérer les besoins de recherche, de reformuler des problématiques, d'agréger des communautés autour de questions communes. Contrairement à l'idée répandue que l'expertise ne crée rien de neuf et se borne à la compilation de données, le travail collectif d'assemblage des connaissances peut réserver des surprises. L'ESCo " Agriculture et biodiversité », par exemple, a montré que si l'impact de l'agriculture sur la biodiversité était bien renseigné, les bénéfices de la biodiversité sur l'agriculture, réputés majeurs, étaient très partiellement étayés par des références scientifiques. Ces acquis pour la recherche sont généralement appréciés par les chercheurs auteurs d'expertise. Ils estiment que leur participation à une ESCo, même si elle est souvent une épreuve dans un emploi du temps chargé, leur a permis de mieux situer leurs travaux dans la diversité des approches, par rapport à d'autres disciplines, par rapport aussi aux besoins des utilisateurs. L'intérêt de l'ouverture à d'autres disciplines est, pour la majorité des interviewés, une motivation forte d'implication dans l'expertise. $\mathrm{C}^{\prime}$ est, $\mathrm{d}$ 'autre part, la rédaction de la synthèse finale qui leur apparaît la plus créative et porteuse de renouvellement scientifique, témoignant de l'utilité de ce travail difficile de construction des éléments de réponse à une question sociétale. Une ESCo représente une forme de pédagogie active de la science en société et, en même temps, une opportunité d'ouverture des chercheurs vers les questions de société.

\section{Quels impacts dans la société ?}

Dès lors qu'elles traitent de questions larges, il est difficile d'évaluer avec précision l'impact des expertises sur les débats et les décisions postérieures à leur diffusion. On peut citer toutefois un exemple avec la commande d'une étude consécutive à l'ESCo " Pesticides, agriculture et environnement » pour évaluer les moyens de réduire l'utilisation des pesticides (étude "Écophyto $\left.R \& D^{4} »\right)$, et l'apport de cette étude à la conception de la stratégie gouvernementale (plan «Écophyto 2018 »).

S'agissant de la recherche, les ESCo ont motivé à l'Inra le développement de programmes de grande envergure sur le comportement alimentaire («Fruits et légumes ») et l'agroécologie (« Agriculture et biodiversité »).

Plus globalement, l'ESCo « Pesticides, agriculture et environnement », et les débats qui l'ont suivie, ont positionné l'Inra en tant qu'institution publique de recherche dans une nouvelle relation à la profession agricole et aux différents acteurs du débat. Cette évolution est confirmée par des ESCo postérieures à celles qui sont citées

\footnotetext{
4 Étude menée par l'Inra à la demande du ministère de l'Écologie, de l'Énergie, du Développement durable et de la Mer et du ministère de l'Alimentation, de l'Agriculture et de la Pêche (Butault, J.P., Dedryver, C.A., Gary, C., Guichard, L., Jacquet, F., Meynard, J.M., Nicot, P., Pitrat, M., Reau, R., Sauphanor, B., Savini, I., Volay, T., 2010. Écophyto RED. Quelles voies pour réduire l'usage des pesticides ? Synthèse du rapport d'étude, Inra, http:/ /www6.paris.inra.fr/depe/Projets/Ecophyto-R-D).
} 
Encadré 4. Évaluations des activités d'ESCo à l'Inra

- Le projet ASIRPA (2011-2013), «Analyse des impacts de la recherche publique agronomique » (sur la société, les politiques publiques, l'environnement...), a inclus $\mathrm{l}^{\prime} \mathrm{ESCo}$ «Pesticides, agriculture et environnement » dans ses objets d'étude ${ }^{5}$.

- Les conditions d'un engagement des compétences scientifiques dans la réalisation des ESCo, rapport d'évaluation commandé à Pierre Stengel par Marion Guillou, P.-D.G. de l'Inra (2012).

- Retours de l'ESCo pour la recherche et pour l'Inra, rapport commandé à Yves Le Bars par la DEPE (2012).

dans ce texte: "Flux d'azote liés aux élevages» et «Variétés végétales tolérantes aux herbicides ».

\section{Éléments de conclusion et de discussion}

Les ESCo sont une modalité d'accomplissement de la mission d'expertise confiée aux établissements publics de recherche français. Elles prennent place dans l'ensemble des nouvelles approches qui s'inventent pour nouer des liens plus étroits entre la recherche et la société et cultiver l'association de l'excellence et de la pertinence.

Leur crédibilité repose sur la confiance dans le processus. Nous en avons évoqué quelques déterminants majeurs : un cadre explicite de principes et de méthode, la rigueur et la transparence dans leur mise en œuvre. Dans ce dispositif, la pluralité des experts, dans ses diverses composantes, apparaît comme le gage principal d'impartialité collective.

La pertinence de l'expertise dépend de la capacité à coconstruire les questions avec les commanditaires et les tenants du débat public, à traduire la commande en questions à la recherche, à assurer que les savoirs nécessaires ont été mobilisés et organisés selon un gradient de robustesse des arguments, et enfin, à garantir que les options décrites sont toujours rapportées à des connaissances publiées.

Le bilan, considéré comme positif à l'issue des évaluations, devrait avoir un caractère cumulatif. Au-delà de la réputation d'un établissement particulier, l'objectif pour la communauté scientifique est de convaincre qu'elle sait extraire de la masse des connaissances disponibles, et communiquer avec le minimum de biais, les éléments pertinents pour débattre des enjeux collectifs. Il s'agit non pas d'imposer l'autorité de la science, mais de maintenir ou de rétablir sa fonction de support indispensable à un débat démocratique qui, sans elle, se résume à un affrontement de convictions et d'intérêts inextricablement mêlés.

Les progrès en ce sens sont dépendants de la mobilisation des compétences existantes. Pour les chercheurs, l'investissement dans l'expertise ne va pas de soi. Parfois refusé dans son principe, il est surtout considéré a priori comme consommateur d'un temps plus utilement consacré à la réalisation des projets de recherche. L'expertise oblige les chercheurs à sortir de leur domaine d'excellence, à accepter parfois une remise en cause de leur approche habituelle et à investir des zones frontières. L'objectif ici n'est pas d'exposer un savoir devant les pairs (cas de la publication scientifique), mais d'ajuster collectivement ce savoir aux besoins d'information de l'action publique, en opérant une forme de transgression contraire aux canons de la pratique de recherche. L'implication des chercheurs pourrait être stimulée par une meilleure intégration des travaux d'expertise dans la dynamique de production des connaissances, une diffusion large des rapports et synthèses des $\mathrm{ESCo}$, dont le statut éditorial aujourd'hui n'est pas de même rang qu'une publication scientifique, et la reconnaissance de la fonction d'expertise dans la carrière du chercheur. Un effort ciblé des institutions de recherche, notamment en matière d'évaluations individuelles et collectives, devrait donc soutenir l'attrait de la fonction d'expert pour les chercheurs.

5 http://www6.inra.fr/asirpa. 